\title{
Comparative Throughput of WiFi \& Ethernet LANs using OPNET MODELER
}

\author{
Er. Ishu Gupta \\ Asst. Prof., \\ RIMT-MAEC, \\ Mandi Gobindgarh
}

\author{
Er. Perminder Kaur \\ Asst. Prof., \\ RIMT-MAEC, \\ Mandi Gobindgarh
}

\begin{abstract}
The comparison between the performance of both wired and wireless networks is based on various performance parameters. The performance of the wired Ethernet is very sensitive to the number of users and the offered load. When the number of users/offered load is high then the collisions increase resulting in larger wastage of the medium and lowering overall throughput. The aim of this research is to compare the throughput of LANs using Ethernet and WiFi under three different scenarios: 25, 50 and 100 users using simulator OPNET-MODELER. By analyzing the graphs, it is concluded that throughput of Wireless LAN (WiFi) is greater than that of Ethernet with operating at transmission speed of $10 \mathrm{Mbps}$ when sufficient number of users are deployed that the model can support. When the number of users is increased beyond the certain limit, throughput decreases. The throughput of Ethernet and WiFi keeps on increasing and decreasing within some specific range due to the propagation delay and overhead collisions affecting at that time.
\end{abstract}

\section{Keywords}

OPNET-MODELER, WiFi, Ethernet, throughput comparison, network simulation.

\section{INTRODUCTION}

Wireless Network refers to any type of computer network that is wireless and is commonly associated with a telecommunications network whose interconnection between nodes is implemented without the use of wires [1]. This network is getting popular nowadays due to easy to set up feature and no cabling required. Wireless LAN (WLAN) technology is gaining broad acceptance and being adopted worldwide in organizations [2].

The most popular form of WLAN is identified as $802.11 \mathrm{~b}$, otherwise known as WiFi. Equipment that follows $802.11 \mathrm{~b}$ standard will be capable of a theoretical data throughput rate of 11 Mbps. This standard is in the same family as Ethernet, generally known by the designation 802.3

\subsection{Introduction to WiFi (802.11b)}

$\mathrm{WiFi}$ - is a short name for Wireless Fidelity. IEEE 802.11 Wi$\mathrm{Fi}$ is oriented towards computer- to-computer connections, as an extension of or substitution for cabled LANs. The aim of the IEEE
802.11 standard is to provide wireless connectivity to devices that require a quick installation, such as portable computers, PDAs, or generally mobile devices inside a WLAN [3].

\subsection{Introduction to Ethernet (802.3)}

Ethernet has been a relatively inexpensive, reasonably fast, and very popular LAN technology for several decades [4]. Ethernet was originally developed by Digital, Intel and Xerox (DIX) and has been designed as a 'broadcast' system, i.e. stations on the network can send messages whenever and wherever it wants. All stations may receive the messages, however only the specific station to which the message is directed will respond [6]. The most commonly installed Ethernet systems are called 10BASE$\mathrm{T}$ and provide transmission speeds upto $10 \mathrm{Mbps}$.

\subsection{Throughput}

In communication of various types of networks, such as Ethernet, network throughput is the average rate of successful message delivery over a communication channel. The throughput is usually measured in bits per second (bit/s or bps) or in data packets per second or data packets per time slot. These data may be delivered over a physical or logical link, or pass through a certain network node [7]. The system throughput or aggregate throughput is the sum of the data rates that are delivered to all terminals in a network.

\section{PERFORMANCE EVALUATION}

The Ethernet is a multi-access network, meaning that a set of nodes sends and receives frames over a shared link. It implements the capability of transmitting and monitoring a connected bus link at a same time. It has full duplex capability [5].

\subsection{Simulation set-up for Ethernet}

Setup for an Ethernet network model operating at a data rate of 10Mbps in a star topology using OPNET Modeler 14.0 with 25 users is as shown in Fig. 1 


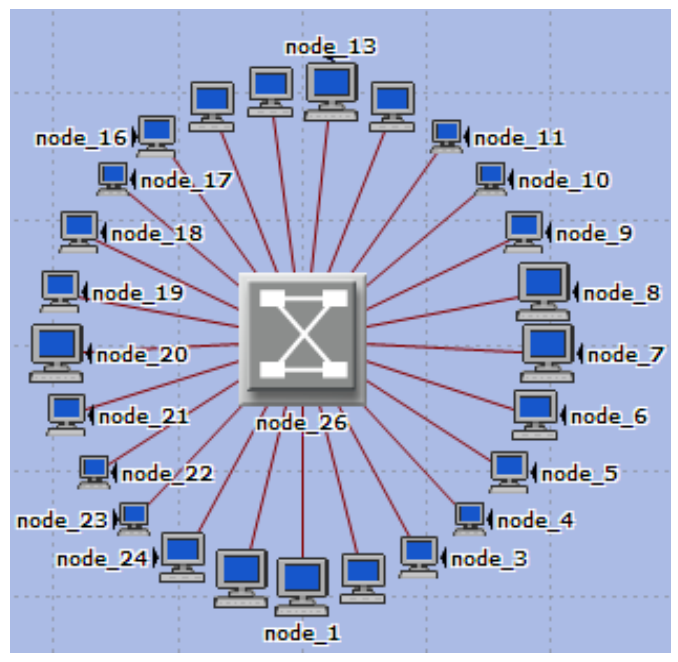

Figure 1: Ethernet network model for 25 Ethernet stations

Similarly this baseline network can be expanded for other two scenarios with 50 and 100 users.

\subsection{Simulation set-up for Wireless LAN}

Wireless LAN (IEEE 802.11) protocol is an alternative to existing local area network access technologies. It allows multiple users within the wireless domain to communicate using a radio connection. The Wireless LAN model suite includes the features of the IEEE 802.11, 802.11b, 802.11a, 802.11g, and 802.11e standards.

Setup for a Wireless network model operating at a data rate of $10 \mathrm{Mbps}$ in a star topology using OPNET Modeler 14.0 is as shown in Fig. 2.

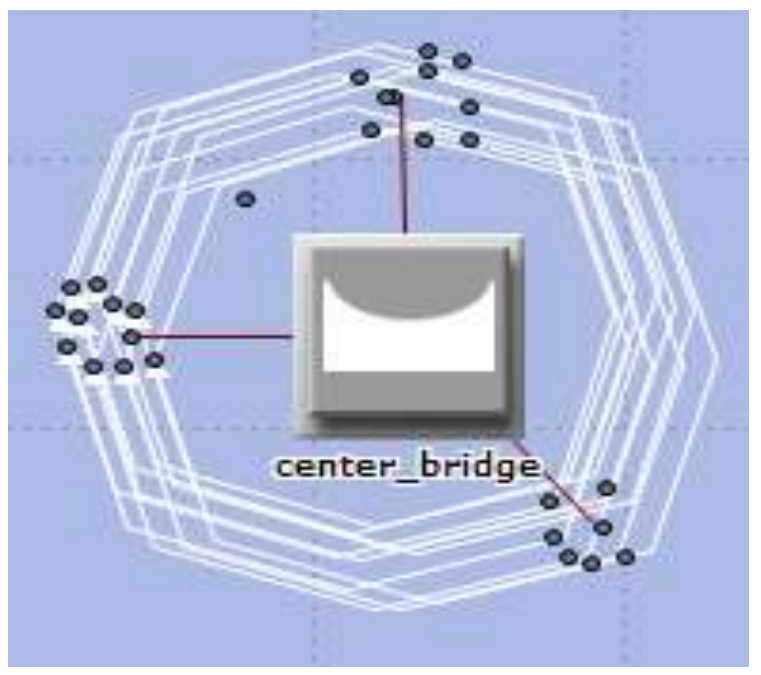

Figure 2: Wireless network model for 25 users

Similarly this baseline network can be expanded for other two scenarios with 50 and 100 users.

\section{PERFORMANCE ANALYSIS}

\subsection{Result analysis for Ethernet}

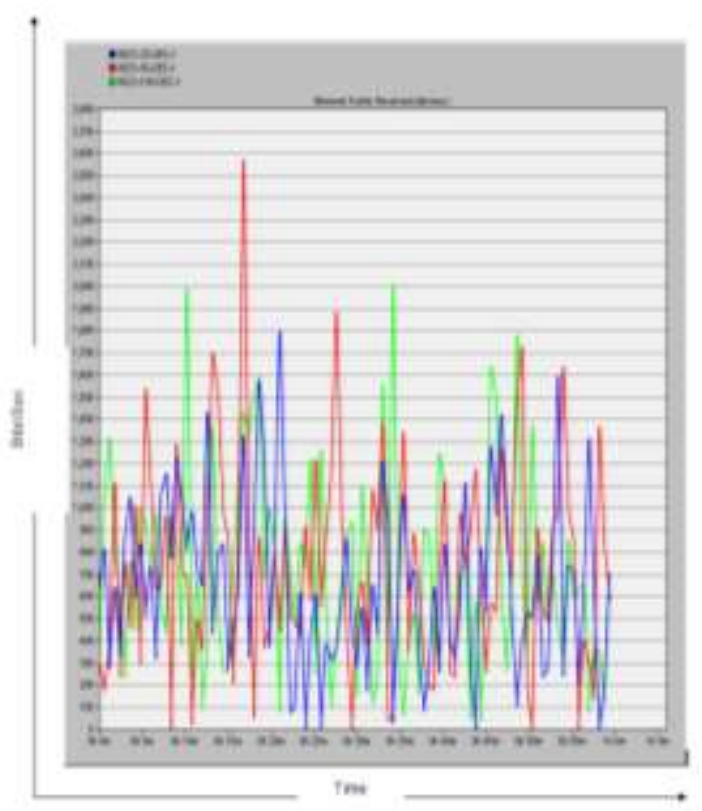

Figure 3: Throughput (bits/sec) of different scenarios on node 20 for Ethernet

In Fig. 3, it is observed that the maximum throughput when 25 users are employed is $1800 \mathrm{bits} / \mathrm{sec}$. In this case, it increases and decreases relative to collision count and traffic at that time. During large traffic, the rate of collision count increases which further affects the throughput of the system. When the number of users is increased to 50, the throughput in that case is maximum. In this LAN, Ethernet64_switch is used which can connect 65 users. So, increasing the number of users will increase the performance and throughput of the network. But when the number of users is increased to 100 , its throughput will be less as compared to that of 50 users because more overhead and delay is encountered.

\subsection{Result analysis for Wireless LAN}

In Fig.4, it is observed that maximum throughput is achieved in the case when less number of users is deployed. When the number of users is increased, the throughput decreases as for wireless systems, these effects associated with wireless transmission limit the SNR (Signal to Noise Ratio) and bandwidth of the received signal, and therefore the maximum number of bits that can be sent. With less number of users, the overall performance of the system increases as data transmission will be faster. 


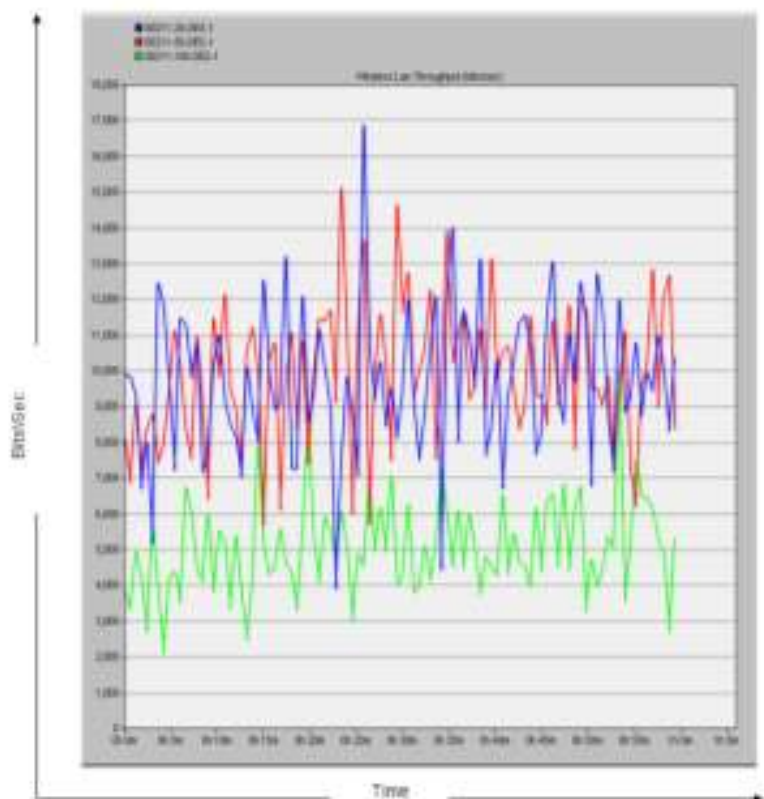

Figure 4 : Throughput (bits/sec) of different scenarios on STA_20 for Wireless LAN

\subsection{Comparing throughput of Ethernet and WiFi}

\subsubsection{Comparison of Scenario-1 with 25 users}

While comparing throughput graph of Ethernet and WiFi in scenario-1 of 25 users on node_10, the graph will appear as shown in Fig. 5. Throughput in bits/sec of Wireless LAN is shown in red colour and of Ethernet in blue colour in the graph.

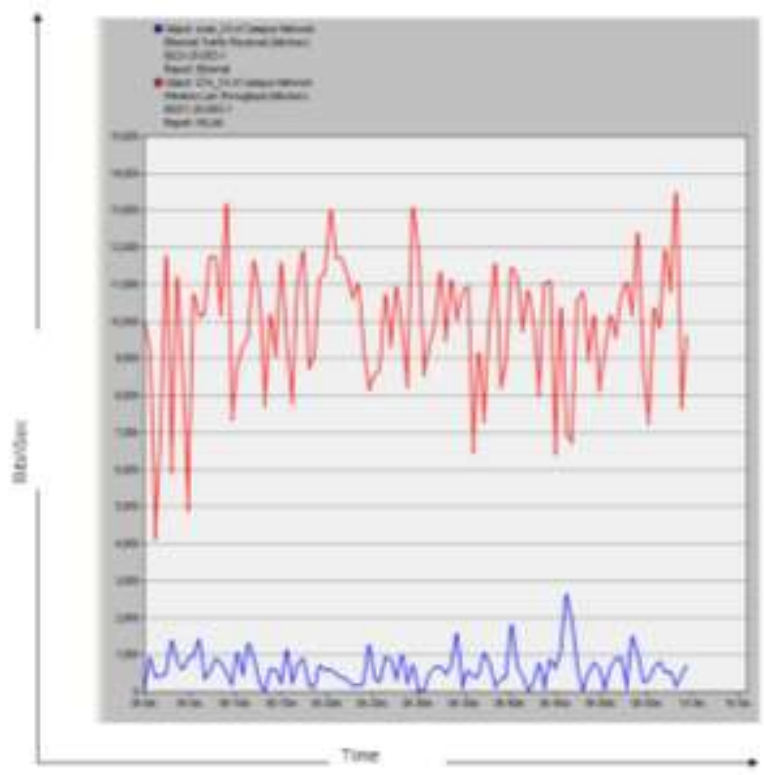

Figure 5: Graph comparing throughput of Ethernet and WiFi on node 10
By analyzing this graph, it is observed that the throughput of Wireless LAN is greater than throughput of Ethernet. The Ethernet throughput begins from the lower value, after that it keeps on increasing and decreasing within some specific range. At the same time, the throughput of Wireless LAN (WiFi) begins with large value. After some time it decreases sharply, and again increase sharply within some specific range. The reason for this increasing and decreasing is propagation delay and overhead collisions affecting at that time.

\subsubsection{Comparison of Scenario-2 with 50 users}

While comparing throughput of Ethernet and $\mathrm{WiFi}$ in scenario-2 of 50 users on node_40, the graph will appear as shown in Fig. 6. Throughput in bits/sec of Wireless LAN is shown in red colour and of Ethernet in blue colour in the graph.

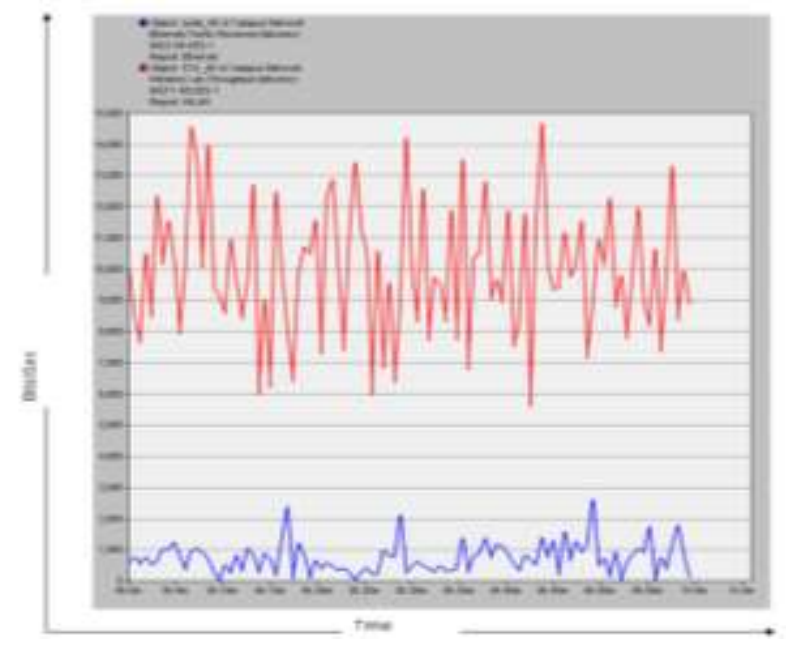

Figure 6: Graph comparing throughput of Ethernet and WiFi on node_40

\subsubsection{Comparison of Scenario-3 with 100 users}

While comparing throughput of Ethernet and WiFi in scenario3 of 100 users on node_60, the graph will appear as shown in Fig. 7 


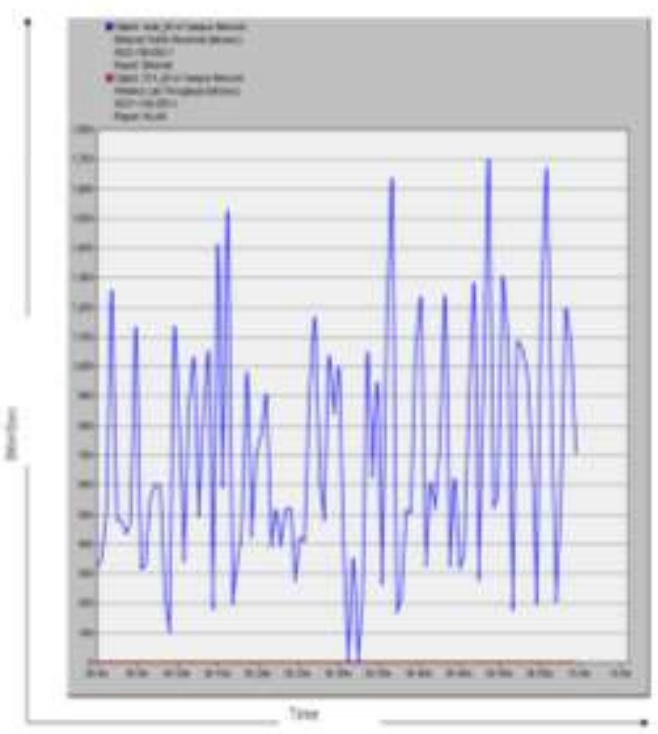

Figure 7: Graph comparing throughput of Ethernet and WiFi on node 60

In this graph, it is observed that by increasing number of users to 100 , the throughput of Ethernet increases and decreases achieving higher values.

\section{RESULTS AND CONCLUSIONS}

By analyzing the graphs, it is observed that throughput of Wireless LAN is greater than that of Ethernet operating at transmission speed of $10 \mathrm{Mbps}$. The Ethernet throughput begins with the small value, after that it keeps on increasing and decreasing within some specific range. At the same time, the throughput of Wireless LAN (WiFi) begins with large value. After some time it decreases sharply, and again increase sharply within some specific range. The reason for this increasing and decreasing is propagation delay and overhead collisions affecting at that time. During large traffic, the rate of collision count increases which further affects the throughput of the system.

In the Ethernet LAN, Ethernet64_switch is used which can connect 65 users. So, throughput of network considering 50 users is best as compared to that of 25 and 100 users. While in case of Wireless LAN, by increasing the number of users, the throughput decreases, all of the effects associated with wireless transmission limit the SNR ( Signal to Noise Ratio) and bandwidth of the received signal, and therefore the maximum number of bits that can be sent. In this case, deploying less no. of users gives more throughputs.

\section{FUTURE WORK}

The future work may be done to compare throughputs using some other wired and wireless protocols. Results may be concluded on various other parameters rather than throughput. Results can be implemented using any other version of Simulator OPNET Modeler and any other Simulator can also be used.

\section{REFERENCES}

[1] Albert Banchs, Xavier Perez-Costa, Daji Qiao,"Providing Throughput Guarantees in IEEE 802.11e Wireless LANs", Proc. IEEE WCNC'02.

[2] Andrew S. Tanenbaum, "Computer Networks", $3^{\text {rd }}$ Edition, Pearson Edu. 2007.

[3] Gent, G. P., Downing, C., Dalton, J.,"Comparative Performance of Wireless and Powerline LANs for Streaming Media", Website at www.citeseerx.ist.psu.edu.

Giuseppe Anastasi, Eleonora Borgia, Marco Conti, Enrico Gregori,"IEEE 802.11b Ad Hoc Networks: Performance Measurements", Journal of Cluster Computing, Springer, vol. 8, 2005.

[5] Jia Wang and Srinivasan Keshav,"Efficient and Accurate Ethernet Simulation", Proc. Of the 24th Conference on Local Computer Networks (LCN'99), pp. 182-191, 1999.

[6] Mahhub Hassan and Leonard Oon,"Performance Comparison of ATM LAN with FDDI and Fast Ethernet", Pacific Telecommunications Council Sixteenth Annual Conference, 1994.

[7] Sanjeev R. Kulkarni, Pramod Viswanath," A deterministic approach to throughput scaling in wireless networks", IEEE Trans. on Information Theory, vol. 50, pp. 1041- 1049, 2004. 